Received Date : 06-Jun-2013

Revised Date : 09-Jul-2013

Accepted Date : 01-Aug-2013

Article type : Original Article

\title{
A DYW-protein knockout in Physcomitrella affects two closely spaced mitochondrial editing sites and causes a severe developmental phenotype
}

Mareike Rüdinger ${ }^{1,2,3}$, Peter Kindgren ${ }^{3}$, Anja Zehrmann ${ }^{4}$, Ian Small ${ }^{3}$ and Volker Knoop ${ }^{1}$

${ }^{1}$ IZMB - Institut für Zelluläre und Molekulare Botanik, Abteilung Molekulare Evolution, Universität Bonn, Kirschallee 1, 53115 Bonn, Germany

${ }^{2}$ Department of Cell Biology, Faculty of Biology, University of Marburg, Karl-von-Frisch-Str. 8, 35043 Marburg, Germany

${ }^{3}$ Australian Research Council Centre of Excellence in Plant Energy Biology, University of Western Australia, Crawley, Western Australia 6009, Australia

${ }^{4}$ Molekulare Botanik, Universität Ulm, 89069 Ulm, Germany

Running head: Assigning all RNA editing factors in moss mitochondria

Key words: Plant mitochondrial RNA editing, Pentatricopeptide repeat proteins, RNA binding code, DYW domain, Cytidine deaminase, Physcomitrella patens

\section{Corresponding author:}

Volker Knoop

volker.knoop@uni-bonn.de

Tel. +49228736466

Fax +49228736466

\section{Abstract}

RNA-binding pentatricopeptide repeat (PPR) proteins carrying a carboxyterminal DYW domain similar to cytidine deaminases have been characterized as site-specific factors for C-

This article has been accepted for publication and undergone full peer review but has not been through the copyediting, typesetting, pagination and proofreading process which may lead to differences between this version and the Version of Record. Please cite this article as an 'Accepted Article', doi: 10.1111/tpj.12304

This article is protected by copyright. All rights reserved. 
to-U RNA editing in plant organelles. Here we report that knockout of DYW-PPR_65 in Physcomitrella patens causes a severe developmental phenotype in the moss and specifically affects two editing sites located 18 nucleotides apart on the mitochondrial $c c m F C$ mRNA. Intriguingly, PPR_71, another DYW-type PPR, had previously been identified as editing factor specifically affecting only the downstream editing site, ccmFCeU122SF. The now characterized PPR_65 specifically binds only to the upstream target site, ccmFCeU103PS, in full agreement with a recent RNA recognition code for PPR arrays. The functional interference between the two editing events may be caused by a combination of three factors: (i) the destabilization of an RNA secondary structure interfering with PPR_71 binding by prior binding of PPR_65, (ii) the resulting upstream C-U conversion or (iii) a direct interaction between the two DYW proteins. Indeed, we find the Physcomitrella DYW-PPRs to interact in yeast-2-hybrid assays. The moss DYW-PPRs also interact yet more strongly with MORF/RIP proteins of Arabidopsis known to be general editing factors in flowering plants, although MORF homologues are entirely absent in the moss. Finally, we demonstrate binding of Physcomitrella DYW-PPR_98, for which no KO lines could be raised, to its predicted target sequence upstream of editing site atp9eU92SL. Together with the functional characterization of DYW-PPR_65, this completes the assignment of RNA editing factors to all editing sites in the Physcomitrella mitochondrial transcriptome.

\section{Introduction}

C-to-U RNA editing of messenger RNAs is a hallmark of gene expression in chloroplasts and mitochondria of land plants. Many cytidines are converted into uridines at specific sites to reconstitute important codon identities encoding phylogenetically conserved amino acids. More than two decades after its discovery (Covello and Gray, 1989; Gualberto et al., 1989; Hiesel et al., 1989) the process of plant organelle RNA editing has remained an 
evolutionary mystery, however. Some plants like Selaginella moellendorffii have thousands of editing sites (Hecht et al., 2011), essentially only serving to repair protein sequence information that is properly encoded in the DNA in others. In the marchantiid liverworts, to give an extreme counter-example, the RNA editing system is completely lost (Groth-Malonek et al., 2007;Rüdinger et al., 2012) and not one editing site has been found in the organelles.

While the reasons for the existence of RNA editing in land plants are still enigmatic, tremendous progress has been made over recent years to understand how specific cytidines are targeted for conversion into uridines. After the original discoveries of the first specific RNA editing factors in Arabidopsis thaliana chloroplasts (Kotera et al., 2005) and mitochondria (Zehrmann et al., 2009), many more such factors have been identified (Chateigner-Boutin and Small, 2010;Chateigner-Boutin and Small, 2011;Finster et al., 2012;Knoop, 2011). All of them turned out to be pentatricopeptide repeat (PPR) proteins encoded by a highly diversified gene family in the plant lineage (Lurin et al., 2004).

Tandem repeat arrays of the PPR motifs mediate sequence-specific binding to RNAs with a 1:1 relationship between motifs and nucleotides and a recognition code assigning specific amino acid identities in PPR motifs to specific RNA nucleotides has been proposed (Barkan et al., 2012; Yagi et al., 2013). The site-specific plant RNA editing factors in particular carry additional domains behind their PPR arrays at the carboxy-termini, minimally an "E" extension and frequently an additional DYW domain. The latter is of particular interest for several reasons. Firstly, a similarity of the DYW domain to cytidine deaminases (Salone et al., 2007) is well supported by bioinformatic structural analyses (Iyer et al., 2011). Secondly, occurrence and diversity of proteins with a DYW domain correlate very well with observed organelle RNA editing frequencies (Rüdinger et al., 2008;Rüdinger et al., 2012).

This article is protected by copyright. All rights reserved. 
Significantly, after an initial discovery of DYW-type PPR proteins outside of land plants in Naegleria gruberi (Knoop and Rüdinger, 2010), mitochondrial RNA editing could indeed be demonstrated in this protist (Rüdinger et al., 2011a). However, direct demonstration of a cytidine deaminase activity mediated by this domain is as yet lacking (Hammani et al., 2009; Nakamura and Sugita, 2008;Okuda et al., 2009). Numerous editing factors identified in flowering plants carry only E/E+ additions and lack a DYW domain, but may recruit the DYW domain in trans (Boussardon et al., 2012). At least one additional family of proteins named MORFs for "Multiple Organellar RNA editing Factors" (Takenaka et al., 2012), alternatively also labelled as RIPs for "RNA editing factor Interacting Proteins" (Bentolila et al., 2012;Bentolila et al., 2013) and a related protein including an RNA-recognition motif (Sun et al., 2013) play important additional roles as general editing factors in the flowering plants.

Other than the dominant model flowering plant Arabidopsis thaliana, the moss Physcomitrella patens has been used as an alternative model organism to investigate RNA editing factors. Physcomitrella perfectly supports the idea of a correlation between DYWtype PPR proteins and RNA editing. Only eleven mitochondrial (Rüdinger et al., 2009) and two chloroplast RNA editing sites (Miyata et al., 2002) match well with only 10 DYW-type PPR proteins in the moss nuclear genome, which neither encodes E/E+ type PPR proteins lacking a DYW domain nor MORF/RIP proteins. Six of these DYW-PPRs have been assigned to nine of the mitochondrial editing sites by knockout studies (Ohtani et al., 2010;Rüdinger et al., 2011b;Tasaki et al., 2010;Uchida et al., 2011).

Here we report data on two of the DYW-PPR proteins in Physcomitrella that have remained functionally unassigned. A knockout of DYW-PPR_65 causes a severe developmental phenotype in the moss, associated with the loss of RNA editing at two

This article is protected by copyright. All rights reserved. 
important sites in the $c c m F C$ mRNA. Interestingly, the second editing site was already assigned to another DYW-type PPR protein (Tasaki et al., 2010) and possible scenarios causing the functional interference of the two proteins are investigated and discussed here.

Whereas no knockout could be raised for DYW-PPR_98, we find that this protein binds well to its predicted RNA target upstream of an editing site in atp 9 , the only remaining RNA editing site in mitochondria of the moss lacking assignment of an editing factor. In essence, this completes the assignment of specific factors to all editing sites in a plant organelle genome, all of which turn out to be DYW-type PPR proteins in the case of mitochondrial RNA editing in Physcomitrella.

\section{Results}

A Physcomitrella patens knockout line was raised for the gene encoding DYW-type PPR_65, which contains 15 PLS-type PPR repeats upstream of a conserved E/E+ and DYW-domain at its carboxy-terminus (Fig. 1A). Obtaining KO plants proved to be difficult, given that this gene knockout turned out to cause very severe retardation in moss development. Tiny moss plants became available for downstream experimentation only after an extended growth period of several months (Fig. 1B). The genetic knockout of PPR_65 in the new KO line could be confirmed at the genome (Fig. 1C) and transcript levels (Fig. 1D).

To check upon growth and development retardation of the PPR_65 KO line in comparison to WT, equal amounts of plant material were used as starting material for dispersion and cultivation on Knop media with and without supplementation of sucrose (Fig. 2A). The mutant grew significantly slower and remained in the protonema stage much longer. However, upon extended incubation in liquid Knop medium supplemented with sucrose, healthy looking leafy gametophores could finally be obtained for the PPR_65 KO line (Fig. 2B), indicating that no developmental program is fundamentally disturbed.

This article is protected by copyright. All rights reserved. 
Assuming that the severe phenotype of the new PPR_65 KO line results from impaired RNA editing, we checked upon all 13 known RNA editing events in Physcomitrella (Fig. 3). Direct sequencing of RT-PCR products showed a complete defect of RNA editing for the two known editing sites in the mitochondrial ccmFC gene (ccmFCeU103PS and ccmFCeU122SF) that is involved in cytochrome c biogenesis. None of the other nine mitochondrial RNA editing sites (Fig. 3) or the only two chloroplast editing sites in P. patens (in the rps14 gene, not shown) were affected. An independent second Physcomitrella patens PPR_65 KO clone was obtained which showed the same characteristics as above, supporting that the severe growth phenotype and the observed editing defect are directly correlated.

To investigate whether the severe developmental retardation observed upon $\mathrm{KO}$ of PPR_65 might be due to a more global influence on the $P$. patens mitochondrial transcriptome we checked the expression of $c c m F C$ in comparison to transcripts of several other loci via real-time quantitative RT-PCR. The transcripts chosen for comparison are also dependent on RNA editing for correct expression and encode proteins involved in different aspects of mitochondrial biogenesis. No reduction in transcript levels was found for the ccmFC transcript, for the mitochondrial 18S rRNA, the mRNAs for nad5 (complex I), cox3 (complex IV), atp9 (complex V) or rps14, encoding ribosomal protein S14 (Suppl. Fig. 1). In fact, all mitochondrial transcripts were found to be slightly increased rather than reduced in abundance in comparison to the nuclear reference genes, likely reflecting a plant cell response attempting to compensate for the mitochondrial defect. We conclude that the severe phenotype of the PPR_65 KO line is a direct consequence of a cytochrome c biogenesis defect caused by lacking editing of the $c c m F C$ transcript and consequently expression of a non-functional CcmFC protein subunit. Notably, the two affected RNA editing sites are highly conserved in many flowering plant taxa whereas pre-edited serine and phenylalanine 
codons are present at the DNA level in the corresponding positions of the mitochondrial ccmFC gene in the alga Chara vulgaris.

Intriguingly, PPR_71, another Physcomitrella patens DYW-type protein, had already been assigned earlier as a specificity factor for ccmFCeU122SF, the downstream site of the two editing sites in $c c m F C$ (Tab. 1). We therefore also used the sensitive PPE method of poisoned primer extension (Chateigner-Boutin and Small, 2007;Peeters and Hanson, 2002) to check for remaining editing of ccmFCeU103PS and ccmFCeU122SF in the new PPR_65 KO line. No remaining partial editing for either site was found in the KO line (Suppl. Fig. 2).

To check whether the KO of PPR_65 may possibly influence the expression of PPR_71, we investigated its transcription in the KO line. Expression of complete PPR_71 transcript was demonstrated in the KO line like in WT (Fig. 1D).

To test for the RNA-binding properties of PPR_65 on the likely target sequences upstream of the two affected editing sites, we used RNA electrophoretic mobility shift assays (REMSAs). To this end we expressed PPR_65 including its complete PPR array and the complete C-terminal domains as a fusion behind the maltose binding protein (MBP) in Escherichia coli Rosetta 2 (DE3) cells. We found that PPR_65 binds with high affinity to its predicted RNA target upstream of ccmFCeU103PS (Fig. 4). In contrast, and independent of a cytidine or uridine being present at the upstream editing position, no significant binding of PPR_65 was seen for the downstream target sequence upstream of ccmFCeU122SF, for which PPR_71 had previously been identified as the specificity factor.

These observations fit very nicely to the proposed PPR recognition code for RNA sequences (Barkan et al., 2012), as can be seen for alignments of the crucial positions 6 and 1' within the pentatricopeptide repeat arrays of PPR_65 and PPR_71 with the sequence This article is protected by copyright. All rights reserved. 
upstream of the two $c c m F C$ editing sites (Fig. 5). The binding prediction for the PPR array of PPR_65 fits very well to the RNA target sequence upstream of ccmFC103PS whereas PPR_71 fits well to the region upstream of ccmFCeU122SF but not vice versa (see Figs. $5 \mathrm{~A}$ and B). Likewise, we also observed good matches between the other RNA editing target sequences and the relevant amino acids of their cognate specificity factors (Figs. $5 \mathrm{C}$ to $\mathrm{H}$ ). Taking the third amino acid of each PPR motif into account (Fujii et al., 2011; Yagi et al., 2013) did not improve RNA sequence matching in the case of the Physcomitrella DYWPPRs.

Obviously, editing of ccmFCeU122SF mediated by PPR_71 is dependent on PPR_65 itself or on the upstream editing event. Both sites have been found to be edited to high degrees in the steady state population of Physcomitrella wild type RNAs (Rüdinger et al., 2009; Tasaki et al., 2010) whereas editing at both positions is completely abolished upon KO of PPR_65 (Fig. 3, suppl. fig. 2). We used a yeast-2-hybrid approach to investigate whether the dependence of PPR_71 on PPR_65 may be due to direct protein-protein interaction (Fig. 6). For these assays also we included PPR_79 as another unrelated Physcomitrella DYWtype protein, for which we were able to raise a $\mathrm{KO}$ line, which specifically affects editing site nad5eU598RC, fully congruent with identical observations elsewhere (Uchida et al., 2011). Indeed, we observed that PPR_65 shows a tendency for protein-protein interactions both with itself and with the other Physcomitrella DYW proteins (Fig. 6B). Moreover, the Physcomitrella DYW proteins also interact surprisingly strongly with most of the MORF proteins (Multiple organellar RNA editing factors) except MORF5 encoded in the flowering plant Arabidopsis thaliana, although no MORF homologues are encoded in the Physcomitrella patens genome. Particularly strong interactions of the Physcomitrella PPR proteins, which are retained also under more stringent conditions, were seen for MORF1 and 
MORF2 (Fig. 6C). These results support the idea of MORFs being a late evolutionary addendum in seed plants acting on more ancient conserved structures within PPR proteins, maybe the PLS arrays themselves or possibly the E/E+ domain of hitherto unclear function.

Other than a direct protein-protein contact between PPR_65 and PPR_71, the better recognition of the ccmFeU122SF target sequence by PPR_71 due to the upstream exchange of $\mathrm{C}$ to $\mathrm{U}$ in the target sequence could be a possible explanation. However, protein-RNA binding assays suggested only a slightly better affinity of PPR_71 to the upstream edited version of the transcript (Tasaki et al., 2010).

Alternatively, RNA secondary structures in the $c c m F C$ transcript may contribute to the dependency of the latter on the former. Indeed, an RNA hairpin may form in the unedited $c c m F C$ mRNA, which places the downstream editing site close to its end and the upstream editing site close to its loop and which would engage the RNA sequence targets of both DYW-PPRs in base-pairing (Fig. 7A). It is possible that RNA binding of PPR_65 prevents formation of such base-pairing or, alternatively, that PPR_65 binding resolves such a secondary structure. One way or the other, this could subsequently allow PPR_71 to access its unfolded target sequence. Additionally, the conversion of the upstream cytidine to uridine may play a role in destabilizing such an RNA secondary structure (Fig. 7B).

We performed a phylogenetic survey across diverse moss taxa to check upon the requirement for editing at the two $c c m F C$ sites (Fig. 8). Both sites are widely conserved among mosses and appear to be very ancient, congruent with their occurrence also in many flowering plants. In the absence of adequate fossil record references, no reliably dated molecular phylogenies exist as yet for mosses. However, our wide taxon sampling including the earliest branching extant moss genera Sphagnum and Takakia likely covers 200-400 
million years of evolution (Fiz-Palacios et al., 2011). The two editing sites appear to be simultaneously lost at least three times independently in early branches of the moss phylogeny (Fig. 8). The downstream editing site ccmFCeU122SF alone was lost due to conversion of the cytidine into a thymidine on DNA level at least twice, in the isolated genus Timmia and within Bryanae. However, in one case we also observed the opposite case in the genus Diphyscium with the upstream site converted to thymidine but the downstream site retained as a cytidine (Fig. 8). It is unclear at present whether the loss of the upstream editing was accompanied by loss of the PPR_65 orthologue in this isolated moss. However, if so, this would strongly support the idea of a destabilized RNA folding upon replacement of the upstream $\mathrm{C}$ with a $\mathrm{U}$ to facilitate PPR_71 binding (Fig. 7B).

After the work on PPR_65 reported here, RNA editing event atp9eU92SL remained the only editing site in Physcomitrella patens mitochondria lacking assignment of an editing factor (Tab. 1). We were unable to raise KO lines for the two remaining DYW-PPRs in Physcomitrella that as yet lack an assignment of functions. Of these, PPR_45 has a very strong prediction for chloroplast localization making it a good candidate for chloroplast editing (Rüdinger et al., 2011b). In contrast, we noted that PPR_98 is a particularly good candidate to be the specific factor for atp9eU92SL editing given its excellent fit to the upstream sequences according to the PPR binding code (Fig. 5H).

In absence of a KO line for PPR_98, we used the MBP-fused PPR_98 expressed in E. coli Rosetta 2 (DE3) cells for REMSA studies to investigate its binding to the potential atp9 target RNA sequence. We observed a shift in RNA electrophoretic mobility with the atp9 RNA probe upon incubation with MBP-PPR_98 (Fig. 9). The binding appears to be specific given that no binding is observed for the unrelated $c c m F C$ target and that MBP alone even at

This article is protected by copyright. All rights reserved. 
high concentrations causes no shift in the atp 9 target. In competition experiments the binding of PPR_98 to the atp9 target is quickly diminished with increasing concentrations of unlabelled RNA (Fig. 9). These data are in perfect agreement with observations elsewhere finding that knock-down of PPR_98 transcripts causes a decrease of atp9eU92SL editing (Sugita et al., 2013 and M. Sugita, Nagoya, Japan, pers. comm.).

\section{Discussion}

Here we have reported on a knockout strain for a DYW-type PPR protein in Physcomitrella patens, PPR_65, which specifically affects two mitochondrial editing sites in the $c c m F C$ transcript. Its effect on the downstream of the two editing sites (ccmFC122SF) is very likely an indirect effect for several reasons. Firstly, strong RNA binding affinity of PPR_65 was seen only for the upstream target ccmFC103PS but not for the downstream one. Secondly, the unrelated DYW-PPR_71 had earlier been identified as specific factor targeting the downstream site (Tasaki et al., 2010) and the observations fit the predictions of RNA sequence targets deduced from the PPR arrays of the two DYW-PPRs (Figs. 5A and B). Thirdly, PPR_71 binding to its recognition sequence may be hindered by an RNA secondary structure in the $c c m F C$ transcript in the region including the two editing sites, which may be resolved by prior binding of PPR_65 and/or the C-to-U conversion of the upstream editing site. The latter possibility is supported by the previously observed better binding of PPR_71 for targets with the upstream site edited (Tasaki et al., 2010).

Notably, we also found evidence for (moderate) protein-protein interactions between PPR_65 and PPR_71, which may play an additional role. We are, however, somewhat careful about this conclusion given that the results of the $\mathrm{Y} 2 \mathrm{H}$ assays are not reciprocally independent of fusing the interaction partners to either the GAL4 DNA binding or the

This article is protected by copyright. All rights reserved. 
activation domain (Fig. 6). Such observations were similarly made for the MORF-PPR interactions (A.Z., Mizuki Takenaka and Axel Brennicke, Ulm, unpubl. observations) and deserve further attention with respect to possible steric incompatibilities in the protein-protein interactions. Further experimental approaches to investigate the PPR_65-PPR_71 interaction should in the future ideally also consider and include their common target, the ccmFC mRNA as a third interaction partner in a possible 3-component system.

Another interesting finding is the strong binding of the P. patens DYW-PPR proteins under study here with MORF/RIP proteins that have been characterized in flowering plants (Bentolila et al., 2012; Takenaka et al., 2012) but which are entirely absent in non-seed plants like Physcomitrella patens and Selaginella moellendorffii. MORF proteins appear to be a late evolutionary gain only in seed plants. We assume that the here observed interactions of Physcomitrella DYW-PPRs and Arabidopsis MORF proteins have no biological significance but rather indicate that the MORF box serves to bind general, pre-existing features of planttype PPR proteins such as the PPR arrays themselves, in full agreement with the observations made for RIP1/MORF8 (Bentolila et al., 2012).

The now complete assignment of eight DYW-type specificity factors to the eleven editing sites in its mitochondrial transcriptome (Fig. 5) and the absence of MORFs, which contribute an interesting but also complicating factor to RNA editing in angiosperms, make Physcomitrella patens a particularly attractive model system to further study the recognition of RNA targets by PPR arrays in vivo. In particular, the DYW-PPR KO lines that do not show such a dramatic phenotype as the one observed here for PPR_65 are of particular value for downstream complementation studies using a wide array of different constructs for complementation. Well-suited examples are the KO of PPR_79 that affects only one site,

This article is protected by copyright. All rights reserved. 
nad5eU598RC (Uchida et al., 2011), and the KO of PPR_78, which affects two mitochondrial editing sites, cox1eU755SL and rps14eU137SL (Rüdinger et al., 2011b).

There is no doubt that some refinements to the PPR recognition code remain necessary to fully explain why certain cytidines but not others are targeted and why mismatches in some PPRs and their respective RNA targets exist and are tolerated. An example of such mismatches is found for PPR_77 where adenine duplets are found in both of its target sites opposite to "ND" amino acid combinations that would rather predict binding of uridines (Fig. 5D). Scanning the Physcomitrella patens mitochondrial genome indeed identifies a transcribed sequence with uridines instead of adenines in the affected positions that could conceptually allow an even better binding of PPR_77. This sequence in the cob transcript (5'-UUUUUGUGUCAUUAUUUGCUUUACCGUUC-3') would place a cytidine in the position for silent editing of a phenylalanine codon (cobeU930FF). This hypothetical editing event has, however, not been identified in the mitochondrial transcriptome studies. As yet unrecognized amino acid positions may well contribute to selecting certain transcript targets in such cases. Given its straightforward reverse genetics, the ease of handling in the laboratory and a likely lesser influence of other factors on RNA editing, Physcomitrella patens will likely be of further help to understand the still enigmatic mechanisms of RNA sequence targeting and $\mathrm{C}$-to- $\mathrm{U}$ editing.

\section{Experimental procedures}

\section{Plant material and growth conditions}

Protonema of Physcomitrella patens (Hedw.) Bruch \& Schimp. strain Gransden 2004 (Rensing et al., 2008) was cultured as described in Rüdinger et al. 2011b. For transformation, protonema cultured in liquid Knop medium adjusted to $\mathrm{pH} 4.5$ was used. Transformed plants

This article is protected by copyright. All rights reserved. 
were grown on solidified (1\% w/v agar) or, continuously shaken, in liquid Knop medium, optionally enriched with $1 \%$ sucrose.

\section{Construction of knockout $(\mathrm{KO})$ cassette and $P$. patens transformation}

Regions of DYW gene DYW-PPR_65 (2779 bp, scaffold_13 position 1781687 - 1778909 ; GenBank accession no. XM_001754397.1) were amplified by PCR to obtain the upstream homologous region (HR1, 596 bp) using primers A (5'- aGGCGCGCCGTCACGTGGACTCTCTTAATCG-3') and B (5' - ttGCGGCCGCTCACTCATCTGATTGAACACCAG$3^{\prime}$ ) and the downstream homologous region (HR2, 728 bp) using primers C (5'-ttGCGGCCGCTGGACTGCTATAATAACAGGTTG-3') and D (5'-aGGCGCGCCGTAAGCTCCATCATCATCAGG-3'), respectively. As selectable marker nptII (neomycin-phosphotransferase), driven by the nos promoter, was used (Rüdinger et al., 2011b) and inserted between HR1 and HR2. For plant transformation, 10-20 $\mu \mathrm{g}$ of purified construct DNA were used for PEG-mediated (polyethyleneglycol) transfer into protoplasts following published procedures (Hohe et al., 2004). Regenerated protoplasts were cultivated on plant antibioticcontaining medium (geneticin $25 \mu \mathrm{g} / \mathrm{ml}$ ) for two rounds of selection.

\section{Detection of KO plants and verification of editing sites}

DNA was prepared from plant material by a simple extraction method (Edwards et al., 1991). RNA was prepared using the Nucleo- Spin RNA Plant kit (Macherey-Nagel, http://www.macherey-nagel. de). RNA was additionally treated with DNase I (Fermentas Life Sciences, http://www.fermentas.com) to remove potential vestiges of DNA. First strand cDNA was synthesized using the RevertAid ${ }^{\mathrm{TM}}$ M-MulV Reverse Transcriptase kit (Fermentas Life Sciences) and $1 \mu \mathrm{M}$ of oligo-dT primer (5'-T23V-3') per assay. For detection of KO plants PCRs with primer pair 65up/65do (5'-ATGGAAATTGTTGAAGCTGGACTGG-3' and 5'-AACATCCCTTTTCTCCATCACTTGC-3') bordering the homologous regions and PCRs with primer 65up and primer K2 (5'-AGCGGGACTCTGGGGTTCGGACTCTAGC- 3') binding in the integrated $n p t I$ gene were performed. See

Rüdinger et al. 2011b for details of PCR amplification assay using Gotaq polymerase (Promega, http://www.promega.com). RT-PCRs using the phusion hot start flex 2x master mix (New England Biolabs, http://www.neb.com) with primers 65up/65do and 71up (5'AAAATCTACACGGCATAATGCG- 3') and 71do (5'-CCAGTAATCGCCGCAAGAGC3') were performed to verify the absence of DYW-PPR_65 transcripts and the presence of DYW-PPR_71 transcripts in KO plants. For verification of RNA editing sites in P. patens

This article is protected by copyright. All rights reserved. 
wildtype, mutant and other moss species, cDNA was synthesized as described above using hexanucleotide random primer mix (10 $\mu \mathrm{M}$ per assay; Carl Roth, http://www.carlroth.de). Different primer pairs bordering regions with editing sites were used for DNA and cDNA assays (the complete primer list is available from the authors upon request). PCR amplification assays were performed as described in Rüdinger et al. 2011b. PCR products were sequenced directly (Eurofins MWG Operon, www.eurofinsgenomics.eu, GATC Biotech AG, http://www. gatc-biotech.com). DNA and cDNA sequences were aligned to the corresponding gene sequences of the annotated mitochondrial genome of $P$. patens (http://www.ncbi.nlm.nih.gov, accession: NC_007945) and chromatograms were analysed with MEGA 5 (Tamura et al., 2011) and Bioedit 7.0.5.3 (Hall, 1999). MEGA 5 was also used for phylogenetic analysis. RNA secondary structure folding was calculated using mfold (Zuker, 2003).

Poisoned primer extension (PPE) assay was performed as described (Chateigner-Boutin and Small, 2007). CcmFC and rps14 regions were amplified from cDNA and DNA with primers ccmFCfor / ccmFCrev (5'-CTAAAAACAAATAGAGCAGATGGTCC-3' / 5'-GGTCTTGCTCTTACCAAAACTATCC-3') and rps14for / rps14rev (5'-ATGTCAAATCAAATTATACGAGATCATAC-3'/ 5'-GATGCTAATTCACGAAAAACTATACGAG-3'), respectively. PCR products served as templates in the extension reaction starting with 5'FAM labeled reverse primers (5FccmFCeU122eUSF_rev-5' -TAAAGAGGTAAAAATAGGTATTATAGTACCATG-3', 5FccmFCeU103PS_rev 5'-ACCATGAGAAAAAGGAGCACCTGTGG-3' and 5Frps14eU137SL_rev 5'-CTGGAACTATTTCTTGGCAACTTAGAC-3'). The reaction was stopped by incorporation of 2',3'-dideoxyguanosine-5' -triphosphate at the position of the editing site for unedited molecules and at the position of the next cytidine for edited molecules.

\section{Expression and purification of MBP-DYW-PPR_65 fusion protein}

The coding regions of DYW-type PPR_65 and DYW-type PPR_98 genes lacking the Nterminal signal part were amplified with primers PPR_65Ppfor (5'-GGGGACAAGTTTGTACAAAAAAGCAGGCTTAGAGAATCTTTATTTTCAGGGCTCTCACGATCAGATGCCAGGG-3') and PPR_65Ppcomprev (5'-GGGGACCACTTTGTACAAGAAAGCTGGGTCTCACCAAAAATCCCCACAAGAACATAC-3') or PPR_98Ppfor (5' - GGGGACAAGTTTGTACAAAAAAGCAGGCTTAGAGAATCTTTATTTTCAGGGCAGGTGTCCGAAAGGGCGATG-3') and PPR_98Ppcomprev (5'- GGGGACCACTTTGTACAAGAAA-

This article is protected by copyright. All rights reserved. 
GCTGGGTCTCACCAAAAGTCTCCACAAGAACAAAC-3'), respectively using phusion hot start flex 2x master mix. The fragments were inserted in the Gateway cloning vector pDondr-Zeo (Invitrogen, http://www.invitrogen.com) and transferred into the overexpression vector pet41K (EMBL, Heidelberg, http://www.EMBL.de) via Gateway cloning. The fusion proteins were expressed by introducing the corresponding plasmids into Rosetta 2 (DE3) cells (Novagen, http://www.merkmillipore.de). Cultures were grown at $37^{\circ} \mathrm{C}$ in growth media (LB, $250 \mathrm{mM} \mathrm{NaCl}, 50$ Tris-HCl, pH 8.0) with $50 \mu \mathrm{M}$ kanamycin and $17 \mu \mathrm{M}$ chloramphenicol to an OD600 of 0.5. Cultures were kept on ice for 20 minutes, before adding $0.5 \mathrm{mM}$ IPTG for induction of expression. Cells were incubated at $180 \mathrm{rpm}$, at $16{ }^{\circ} \mathrm{C}$ over night and harvested the next day. Pellets were dissolved in ice-cold lysis buffer $(50 \mathrm{mM}$ Tris- $\mathrm{HCl}, 500 \mathrm{mM} \mathrm{NaCl}$, $1 \%$ glycerol, $0.01 \%$ CHAPS, $7 \mathrm{mM} \beta$-mercaptoethanol and 1 Proteinase inhibitor tablet $/ 50 \mathrm{ml}$ [Sigma-Aldrich, http://www.sigmaaldrich.com], pH 8.0) and cells were lysed with a homogenizer. The lysate was cleared by centrifugation at $10000 \mathrm{~g}$ for $10 \mathrm{~min}$. For purification, the cleared lysate was incubated with Ni-NTA agarose for 60 minutes at $4{ }^{\circ} \mathrm{C}$. After 2-4 wash steps with wash buffer (50 mM Tris-HCl, $0.5 \mathrm{M} \mathrm{NaCl}, 20 \mathrm{mM}$ imidazol) protein was eluted in elution buffer $(50 \mathrm{mM}$ Tris- $\mathrm{HCl}, 0.5 \mathrm{M} \mathrm{NaCl}, 250 \mathrm{mM}$ imidazol). Purified recombinant protein was concentrated using a Vivaspin 20 column (GE Healthcare Lifescience, http://www.gelifescience.com) and dialysed into dialysis buffer (50 mM Tris$\mathrm{HCl}, 500 \mathrm{mM} \mathrm{NaCl}, 0.01 \%$ CHAPS, $7 \mathrm{mM}$ ß-mercaptoethanol, $50 \%$ glycerol). Purified protein showed an A260/280 ratio of 0.6, which indicates no or low contamination of nucleic acids. Aliquots were stored at $-70^{\circ} \mathrm{C}$.

\section{RNA electromobility shift assays}

3'-fluorescein-labelled probes (Sigma-Aldrich) were appropriately diluted with RNAse-free water. Prior to the binding reaction RNA was heated to $94^{\circ} \mathrm{C}$ for 2 minutes and incubated on ice for 4 minutes. Binding reaction included $10 \mu \mathrm{l}$ RNA, $5 \mu \mathrm{l}$ protein and $10 \mu \mathrm{l} 2 \mathrm{x}$ buffer (50 $\mathrm{mM}$ Tris- $\mathrm{HCl}, \mathrm{pH} 8.0,100 \mu \mathrm{g} / \mathrm{ml}$ BSA, $4 \mathrm{mM}$ DTT, $1 \mathrm{mg} / \mathrm{ml}$ heparin, RNAseOUT [8 Units/reaction], $200 \mathrm{mM} \mathrm{NaCl}$ ) and was incubated for 30 minutes at $25{ }^{\circ} \mathrm{C}$ in the dark. For competitor experiments, the protein was incubated with the unlabelled RNA in binding buffer for 10 minutes at $25^{\circ} \mathrm{C}$ prior to the probe being added. Samples were applied to $6 \%$ native polyacrylamide gel in THE buffer (170 mM Tris, $330 \mathrm{mM}$ HEPES, $0.5 \mathrm{mM}$ EDTA, pH 8.0) and the results were imaged with a Typhoon Trio imager (GE Healthcare Lifescience).

This article is protected by copyright. All rights reserved. 


\section{Real-time quantitative RT-PCR to compare the expression of mitochondrial genes in wildtype versus KO_65 plants}

RNA was extracted from same amount of wildtype and mutant plant material grown under same conditions and reverse transcription was performed as described above using hexanucleotide random primer mix. Quantitative RT-PCR was performed with SYBR green master mix (Applied Biosystems, Invitrogen, http://www.invitrogen.com) and an amount of cDNA corresponding to $5 \mathrm{ng}$ initial total RNA per $20 \mu \mathrm{l}$ assay on an Applied Biosystems real time PCR system (Applied Biosystems, Invitrogen) with the following program: $95^{\circ} \mathrm{C}$ for 10 minutes (Holding stage), followed by 40 cycles with $95^{\circ} \mathrm{C}$ for $15 \mathrm{sec}$ and $60^{\circ} \mathrm{C}$ for 1 minute (Cycling stage) and finishing with the melting stage $\left(95^{\circ} \mathrm{C}\right.$ for $15 \mathrm{sec}, 60^{\circ} \mathrm{C}$ for $1 \mathrm{~min}$ increasing in $0.3 \mathrm{~K}$ steps up to $95^{\circ} \mathrm{C}$ for $15 \mathrm{sec}$ ). Physcomitrella patens nuclear rrn18 (NCBI Genebank accession no.: X80986) and Phypa_173694 (NCBI Genbank accession no.:XM_001785861.1) were used as reference genes for normalisation. The different primer pairs used to amplify parts of the mitochondrial and reference transcripts are available from the authors upon request. Each sample was run in triplicates and three biologically replicated PCR experiments were carried out. Data were analyzed using the StepOne Software v2.1 (Applied Biosystems, Invitrogen, 2009). To check for product specificity, melting curve analysis was routinely performed.

\section{Yeast two-hybrid assays}

Coding regions (lacking the N-terminal localization signal) of DYW-PPR_65, 71 and 79 were amplified with primers NdeI_65Y2H_for (5'-catcatatgTCTCACGATCAGATGCCAGG-3') and 65Y2H_SmaI_rev (5'-TCCCGGGTCACCAAAAATCCCCACAAGAACATAC-3'), EcoRI_79Y2H_for (5'-cagaattcATGATCCCCACCGGCAAG-3') and 79Y2H_BamHI_rev (5'-GAGGATCCTTACCAGTAATCACCGCAAGAGCAC-3') and EcoRI_71Y2H_for (5'-cagaattcCGACGGGTTGGAGCAAATGATG-3') and 71Y2H_BamHI_rev (5'-GAGGATCCTCACCAGTAATCGCCGCAAGAGC-3'), respectively, using phusion hot start flex 2x master mix as described above. They were cloned both $\mathrm{N}$-terminally in fusion to the GAL4 activator and alternatively to the GAL4 DNA-binding domain using bait vector pGADT7 and prey vector pGBKT7 (CLONTECH Laboratories, http://www.clontech.com), respectively. Interaction studies in yeast cells were performed with the Matchmaker ${ }^{\mathrm{TM}}$ GAL4 Two-Hybrid System 3 (Clontech Laboratories). Different combinations of the resulting bait and prey vectors were introduced simultaneously into yeast cells (PJ69-4A) according to the protocol. In addition, yeast cells were co-transfected with combinations of vectors containing

This article is protected by copyright. All rights reserved. 
the Physcomitrella patens PPR proteins and the nine different Arabidopsis MORF proteins (Takenaka et al., 2012), respectively. As positive control combinations of vectors pGBKT7 p35 and pGADT7T and as negative control combinations of pGBKT7 Lam and pGADT7 T were used. Synthetic drop-out medium without Leu and Trp was inoculated with cells that had incorporated both bait and prey vectors. The overnight cultures were diluted to an $\mathrm{OD}_{600}$ of 0.3 and $5 \mu 1$ thereof dropped on synthetic drop-out plates lacking either Leu and Trp or Leu, Trp, His and Ade. Cells were dropped on the latter plates with $2.5 \mathrm{mM} 3$-aminotriazol added to display interactions with higher stringency (although the employed yeast strain PJ69-4A as a derivative AH109 is not supposed to require the 3-AT according to the manufacturer's protocol). All pGADT7-MORF constructs were tested in parallel yielding no evidence for auto-activation. Growth on the plates was followed up for 10 days.

\section{Acknowledgement}

We wish to thank Clement Boussardon (Perth, Australia) for help with the PPE experiments, Catherine Colas des Francs-Small (ibid.) and Sebastian Hanke (Marburg, Germany) for helpful advice on performing the qRT-PCR experiments and Bianca Wolf (Ulm, Germany) for expert technical assistance with the $\mathrm{Y} 2 \mathrm{H}$ experiments. We are also very grateful to Prof. Stefan A. Rensing (Marburg, Germany) for recommendation of thioredoxin (Phypa_173694) as an internal control for qRT-PCR with P. patens and Prof. Mamoru Sugita (Nagoya University) for providing information on unpublished results Work in the corresponding author's laboratory on Physcomitrella patens RNA editing and DYW-proteins was supported by DFG grant Kn411/7. Research of M.R. in the laboratory of I.S. was supported by a DAAD short-term postdoctoral fellowship. Work at UWA was supported by ARC grant DP120102870 and P.K. is supported through a post-doctoral fellowship from the WennerGren foundation. The authors declare no conflicts of interests.

\section{Reference List}

1. Barkan, A., Rojas, M., Fujii, S., Yap, A., Chong, Y.S., Bond, C.S., and Small, I. (2012) A combinatorial amino acid code for RNA recognition by pentatricopeptide repeat proteins. PLoS Genet. 8, e1002910.

2. Bentolila, S., Heller, W.P., Sun, T., Babina, A.M., Friso, G., van Wijk, K.J., and Hanson, M.R. (2012) RIP1, a member of an Arabidopsis protein family, interacts with the protein RARE1 and broadly affects RNA editing. Proc. Natl. Acad. Sci. U. S. A.

This article is protected by copyright. All rights reserved. 
3. Bentolila, S., Oh, J., Hanson, M.R., and Bukowski, R. (2013) Comprehensive High-Resolution Analysis of the Role of an Arabidopsis Gene Family in RNA Editing. PLoS Genet 9, e1003584.

4. Boussardon, C., Salone, V., Avon, A., Berthome, R., Hammani, K., Okuda, K., Shikanai, T., Small, I., and Lurin, C. (2012) Two interacting proteins are necessary for the editing of the ndhD-1 Site in Arabidopsis plastids. Plant Cell.

5. Chateigner-Boutin, A. L. and Small, I. (2007) A rapid high-throughput method for the detection and quantification of RNA editing based on high-resolution melting of amplicons. Nucl. Acids Res. 35, e114.

6. Chateigner-Boutin, A. L. and Small, I. (2010) Plant RNA editing. RNA Biol. 7, 213 219.

7. Chateigner-Boutin, A. L. and Small, I. (2011) Organellar RNA editing. Wiley. Interdiscip. Rev. RNA 2, 493-506.

8. Covello, P. S. and Gray, M.W. (1989) RNA editing in plant mitochondria. Nature 341, 662-666.

9. Edwards, K., Johnstone, C., and Thompson, C. (1991) A simple and rapid method for the preparation of plant genomic DNA for PCR analysis. Nucl. Acids Res. 19, 1349.

10. Finster, S., J. Legen, Y. Qu, and C. Schmitz-Linneweber. (2012) Land plant RNA editing or: don't be fooled by plant organellar DNA sequences, p. 293-321. In: R. Bock and V. Knoop (eds.), Genomics of Chloroplasts and Mitochondria. Springer, Dordrecht.

11. Fiz-Palacios, O., Schneider, H., Heinrichs, J., and Savolainen, V. (2011) Diversification of land plants: insights from a family-level phylogenetic analysis. BMC Evol. Biol 11, 341.

12. Fujii, S., Bond, C.S., and Small, I.D. (2011) Selection patterns on restorer-like genes reveal a conflict between nuclear and mitochondrial genomes throughout angiosperm evolution. Proc. Natl. Acad. Sci. U. S. A 108, 1723-1728.

13. Groth-Malonek, M., Wahrmund, U., Polsakiewicz, M., and Knoop, V. (2007) Evolution of a pseudogene: Exclusive survival of a functional mitochondrial $\operatorname{nad} 7$ gene supports Haplomitrium as the earliest liverwort lineage and proposes a secondary loss of RNA editing in Marchantiidae. Mol. Biol. Evol. 24, 1068-1074.

14. Gualberto, J. M., Lamattina, L., Bonnard, G., Weil, J.H., and Grienenberger, J.M. (1989) RNA editing in wheat mitochondria results in the conservation of protein sequences. Nature 341, 660-662.

15. Hall, T. A. (1999) BioEdit: a user-friendly biological sequence alignment editor and analysis program for Windows 95/98/NT. Nucl. Acids. Symp. Ser. 41, 95-98.

This article is protected by copyright. All rights reserved. 
16. Hammani, K., Okuda, K., Tanz, S.K., Chateigner-Boutin, A.L., Shikanai, T., and Small, I. (2009) A study of new Arabidopsis chloroplast RNA editing mutants reveals general features of editing factors and their target sites. Plant Cell 21, 3686-3699.

17. Hecht, J., Grewe, F., and Knoop, V. (2011) Extreme RNA editing in coding islands and abundant microsatellites in repeat sequences of Selaginella moellendorffii mitochondria: the root of frequent plant mtDNA recombination in early tracheophytes. Genome Biol Evol 3, 344-358.

18. Hiesel, R., Wissinger, B., Schuster, W., and Brennicke, A. (1989) RNA editing in plant mitochondria. Science 246, 1632-1634.

19. Hohe, A., Egener, T., Lucht, J.M., Holtorf, H., Reinhard, C., Schween, G., and Reski, R. (2004) An improved and highly standardised transformation procedure allows efficient production of single and multiple targeted gene-knockouts in a moss, Physcomitrella patens. Curr. Genet. 44, 339-347.

20. Ichinose, M., Tasaki, E., Sugita, C., and Sugita, M. (2011) A PPR-DYW protein is required for splicing of a group II intron of coxl pre-mRNA in Physcomitrella patens. Plant J.

21. Iyer, L. M., Zhang, D., Rogozin, I.B., and Aravind, L. (2011) Evolution of the deaminase fold and multiple origins of eukaryotic editing and mutagenic nucleic acid deaminases from bacterial toxin systems. Nucl. Acids Res. 39, 9473-9497.

22. Knoop, V. (2011) When you can't trust the DNA: RNA editing changes transcript sequences. Cell. Mol. Life Sci. 68, 567-586.

23. Knoop, V. and Rüdinger, M. (2010) DYW-type PPR proteins in a heterolobosean protist: Plant RNA editing factors involved in an ancient horizontal gene transfer? FEBS Lett. 584, 4287-4291.

24. Kotera, E., Tasaka, M., and Shikanai, T. (2005) A pentatricopeptide repeat protein is essential for RNA editing in chloroplasts. Nature 433, 326-330.

25. Lurin, C., Andrés, C., Aubourg, S., Bellaoui, M., Bitton, F., Bruyère, C., Caboche, M., Debast, C., Gualberto, J., Hoffmann, B., Lecharny, A., Le Ret, M., Martin-Magniette, M.L., Mireau, H., Peeters, N., Renou, J.P., Szurek, B., Taconnat, L., and Small, I. (2004) Genome-wide analysis of Arabidopsis pentatricopeptide repeat proteins reveals their essential role in organelle biogenesis. Plant Cell 16, 2089-2103.

26. Miyata, Y., Sugiura, C., Kobayashi, Y., Hagiwara, M., and Sugita, M. (2002) Chloroplast ribosomal S14 protein transcript is edited to create a translation initiation codon in the moss Physcomitrella patens. Biochim. Biophys. Acta 1576, 346-349.

27. Nakamura, T. and Sugita, M. (2008) A conserved DYW domain of the pentatricopeptide repeat protein possesses a novel endoribonuclease activity. FEBS Lett. 582, 4163-4168.

28. Ohtani, S., Ichinose, M., Tasaki, E., Aoki, Y., Komura, Y., and Sugita, M. (2010) Targeted gene disruption identifies three PPR-DYW proteins involved in RNA editing

This article is protected by copyright. All rights reserved. 
for five editing sites of the moss mitochondrial transcripts. Plant Cell Physiol. 51, 1942-1949.

29. Okuda, K., Chateigner-Boutin, A.L., Nakamura, T., Delannoy, E., Sugita, M., Myouga, F., Motohashi, R., Shinozaki, K., Small, I., and Shikanai, T. (2009) Pentatricopeptide repeat proteins with the DYW motif have distinct molecular functions in RNA Editing and RNA cleavage in Arabidopsis chloroplasts. Plant Cell 21, 146-156.

30. Peeters, N. M. and Hanson, M.R. (2002) Transcript abundance supercedes editing efficiency as a factor in developmental variation of chloroplast gene expression. RNA 8, 497-511.

31. Rensing, S. A., Lang, D., Zimmer, A.D., Terry, A., Salamov, A., Shapiro, H., Nishiyama, T., Perroud, P.F., Lindquist, E.A., Kamisugi, Y., Tanahashi, T., Sakakibara, K., Fujita, T., Oishi, K., Shin, I., Kuroki, Y., Toyoda, A., Suzuki, Y., Hashimoto, S., Yamaguchi, K., Sugano, S., Kohara, Y., Fujiyama, A., Anterola, A., Aoki, S., Ashton, N., Barbazuk, W.B., Barker, E., Bennetzen, J.L., Blankenship, R., Cho, S.H., Dutcher, S.K., Estelle, M., Fawcett, J.A., Gundlach, H., Hanada, K., Heyl, A., Hicks, K.A., Hughes, J., Lohr, M., Mayer, K., Melkozernov, A., Murata, T., Nelson, D.R., Pils, B., Prigge, M., Reiss, B., Renner, T., Rombauts, S., Rushton, P.J., Sanderfoot, A., Schween, G., Shiu, S.H., Stueber, K., Theodoulou, F.L., Tu, H., van de Peer, Y., Verrier, P.J., Waters, E., Wood, A., Yang, L., Cove, D., Cuming, A.C., Hasebe, M., Lucas, S., Mishler, B.D., Reski, R., Grigoriev, I.V., Quatrano, R.S., and Boore, J.L. (2008) The Physcomitrella genome reveals evolutionary insights into the conquest of land by plants. Science 319, 64-69.

32. Rüdinger, M., Fritz-Laylin, L., Polsakiewicz, M., and Knoop, V. (2011a) Planttype mitochondrial RNA editing in the protist Naegleria gruberi. RNA 17, 2058-2062.

33. Rüdinger, M., Funk, H.T., Rensing, S.A., Maier, U.G., and Knoop, V. (2009) RNA editing: only eleven sites are present in the Physcomitrella patens mitochondrial transcriptome and a universal nomenclature proposal. Mol. Genet. Genom. 281, 473481.

34. Rüdinger, M., Polsakiewicz, M., and Knoop, V. (2008) Organellar RNA editing and plant-specific extensions of pentatricopeptide repeat (PPR) proteins in jungermanniid but not in marchantiid liverworts. Mol. Biol. Evol. 25, 1405-1414.

35. Rüdinger, M., Szövényi, P., Rensing, S.A., and Knoop, V. (2011b) Assigning DYW-type PPR proteins to RNA editing sites in the funariid mosses Physcomitrella patens and Funaria hygrometrica. Plant J. 67, 370-380.

36. Rüdinger, M., Volkmar, U., Lenz, H., Groth-Malonek, M., and Knoop, V. (2012) Nuclear DYW-type PPR gene families diversify with increasing RNA editing frequencies in liverwort and moss mitochondria. J. Mol. Evol. 74, 37-51.

37. Salone, V., Rüdinger, M., Polsakiewicz, M., Hoffmann, B., Groth-Malonek, M., Szurek, B., Small, I., Knoop, V., and Lurin, C. (2007) A hypothesis on the identification of the editing enzyme in plant organelles. FEBS Lett. 581, 4132-4138.

This article is protected by copyright. All rights reserved. 
38. Sugita, M., Ichinose, M., Ide, M., and Sugita, C. (2013) Architecture of the PPR gene family in the moss Physcomitrella patens. RNA Biol 10.

39. Sun, T., Germain, A., Giloteaux, L., Hammani, K., Barkan, A., Hanson, M.R., and Bentolila, S. (2013) An RNA recognition motif-containing protein is required for plastid RNA editing in Arabidopsis and maize. Proc. Natl. Acad. Sci. U. S. A 110, E1169-E1178.

40. Takenaka, M., Zehrmann, A., Verbitskiy, D., Kugelmann, M., Härtel, B., and Brennicke, A. (2012) Multiple organellar RNA editing factor (MORF) family proteins are required for RNA editing in mitochondria and plastids of plants. Proc. Natl. Acad. Sci. U. S. A 109, 5104-5109.

41. Tamura, K., Peterson, D., Peterson, N., Stecher, G., Nei, M., and Kumar, S. (2011) MEGA5: molecular evolutionary genetics analysis using maximum likelihood, evolutionary distance, and maximum parsimony methods. Mol. Biol Evol. 28, 27312739.

42. Tasaki, E., Hattori, M., and Sugita, M. (2010) The moss pentatricopeptide repeat protein with a DYW domain is responsible for RNA editing of mitochondrial $c c m F c$ transcript. Plant J. 62, 560-570.

43. Uchida, M., Ohtani, S., Ichinose, M., Sugita, C., and Sugita, M. (2011) The PPRDYW proteins are required for RNA editing of rps 14 , cox 1 and nad5 transcripts in Physcomitrella patens mitochondria. FEBS Lett. 585, 2367-2371.

44. Wahrmund, U., Quandt, D., and Knoop, V. (2010) The phylogeny of mosses addressing open issues with a new mitochondrial locus: group I intron cobi420. Mol. Phylogenet. Evol. 54, 417-426.

45. Yagi, Y., Hayashi, S., Kobayashi, K., Hirayama, T., and Nakamura, T. (2013) Elucidation of the RNA recognition code for pentatricopeptide repeat proteins involved in organelle RNA editing in plants. PLOS ONE 8, e57286.

46. Zehrmann, A., Verbitskiy, D., van der Merwe, J.A., Brennicke, A., and Takenaka, M. (2009) A DYW domain-containing pentatricopeptide repeat protein is required for RNA editing at multiple sites in mitochondria of Arabidopsis thaliana. Plant Cell 21, 558-567.

47. Zuker, M. (2003) Mfold web server for nucleic acid folding and hybridization prediction. Nucleic Acids Res. 31, 3406-3415.

Table 1. Assignment of DYW-type PPR proteins and editing events in Physcomitrella patens

\begin{tabular}{|l|c|c|c|c|c|}
\hline DYW-PPR & PHYPADRAFT & Size $^{*}$ & editing site(s) & comment & study \\
\hline PPR_43 & 154890 & 1073 aa & $\begin{array}{c}\text { None, cox1 intron } \\
\text { splicing factor -- }\end{array}$ & $\begin{array}{c}\text { KO study } \\
\text { Defect DYW domain. }\end{array}$ & (Ichinose et al., 2011) \\
\hline PPR_45 & 156474 & 1097 aa & -- & chloroplast prediction & - \\
\hline PPR_56 & 169414 & 859 aa & $\begin{array}{c}\text { nad3eU230SL } \\
\text { nad4eU272SL }\end{array}$ & KO study & (Ohtani et al., 2010) \\
\hline
\end{tabular}

This article is protected by copyright. All rights reserved. 


\begin{tabular}{|l|c|c|c|c|c|}
\hline PPR_65 & 175641 & 772 aa & ccmFCeU103PS & KO study & This work \\
\hline PPR_71 & 181369 & 833 aa & ccmFCeU122SF & $\begin{array}{c}\text { KO study } \\
\text { Dependent on PPR_65 }\end{array}$ & (Tasaki et al., 2010) \\
\hline PPR_77 & 191892 & 1160 aa & $\begin{array}{c}\text { cox2eU370RW } \\
\text { cox3eU733RW }\end{array}$ & KO study & (Ohtani et al., 2010) \\
\hline PPR_78 & 192620 & 1077 aa & $\begin{array}{c}\text { cox1eU755SL } \\
\text { rps14eU137SL }\end{array}$ & KO study & $\begin{array}{c}\text { (Rüdinger et al., } \\
\text { 2011b) }\end{array}$ \\
\hline PPR_79 & 192787 & 840 aa & nad5eU598RC & KO study & (Uchida et al., 2011) \\
\hline PPR_91 & 211228 & 890 aa & nad5eU730RW & KO study & (Ohtani et al., 2010) \\
\hline PPR_98 & 224041 & 986 aa & atp9eU92SL & protein binding & This work \\
\hline
\end{tabular}

*Sizes differ from Phypadraft models where transcript analyses have identified an alternative gene structure (alternative splice sites or start codons)

\section{Figure Legends}

\section{Figure 1. Knockout of DYW protein gene DYW-PPR_65.}

A. DYW-PPR_65 is a typical plant-type PPR protein of 722 amino acids with an array of 15 PLS-type PPRs (orange, brown and yellow boxes, respectively) followed by carboxyterminal E/E+ and DYW domains (green and blue boxes, respectively). The PPR_65 coding sequence extends over $2779 \mathrm{bp}$ long containing one intron in the N-terminal region (150 bp) and one within the PPR motif stretch (313 bp) correcting the corresponding Physcomitrella patens protein model PHYPADRAFT_175461 (Genbank entry: XP_001754449). Homologous recombination was used to insert the selectable nptII marker cassette into PPR_65 upon protoplast transformation. Binding sites of specific primers used to analyze DNA and cDNA in mutant vs. wildtype (WT) are indicated. B. The growth retardation phenotype of a PPR_65 KO plant line is obvious in comparison to wild type after protoplast regeneration and growth on Knop medium for seven months. C. Expected PCR results are obtained for DNA from the PPR_65 KO line in comparison to WT. Lengths of amplicons between primers 65up and 65do (WT) or alternatively between 65up and K2 targeting the inserted nptII cassette in the KO line are 1552 bp or 1055 bp, respectively. D. Genetic inactivation of PPR_65 in the KO line is demonstrated by RT-PCR with primers 65up and $65 \mathrm{do}$, which results in the expected product of $1239 \mathrm{bp}$ for splicing of the $2^{\text {nd }}$ intron in WT only. RT-PCR alternatively targeting the PPR_71 locus (PHYPADRAFT_181369) with primers 71up and 71do was chosen as a positive reference control resulting in amplification of a 2516 bp product as expected both in WT and the PPR_65 KO line. Negative controls omitting the reverse transcriptase step (-RT) are shown in the respective rightmost lanes.

This article is protected by copyright. All rights reserved. 


\section{Figure 2. Growth and developmental retardation of PPR_65 KO plants.}

A. Equal amounts (30 mg fresh weight) of wild-type and PPR_65 KO plant material were dispersed and transferred to $50 \mathrm{ml}$ of Knop medium optionally supplemented with $1 \%$ sucrose. One $\mathrm{ml}$ of each suspension was spread on corresponding Knop medium plates and incubated for 21 days, which makes the retarded growth and development of the PPR_65 KO line apparent, independent of the addition of sugar in the media. Whereas WT plants have developed leafy structures, KO plants show only protonema structures. B. PPR_65 KO plants grown for 10 weeks on solid media were transferred into Knop liquid medium supplemented with $1 \%$ of sucrose. After extended cultivation in liquid media for 4 weeks, leafy gametophyte structures and gametophores developed and could be transferred to solid medium with $1 \%$ of sucrose and kept for 4 months.

\section{Figure 3. RNA editing defects in the DYW-PPR_65 KO line.}

A. RNA editing at the two known sites in the mitochondrial $c c m F C$ transcript (ccmFCeU103PS and ccmFCeU122SF) is completely lost in the Physcomitrella patens PPR_65 KO mutant as becomes obvious in comparing the cDNA sequencing electropherograms. B. All of the other nine editing sites in the mitochondrial transcriptome of $P$. patens remain unaffected. RNA editing site nomenclature indicates affected gene, position in the reading frame and the resulting amino acid codon change induced by RNA editing (Rüdinger et al., 2009), e.g. proline to serine and serine to phenylalanine in the case of the two RNA editing sites in the $c c m F C$ mRNA. Edited site rps14eU137SL was found to be only edited in $60-70 \%$ of cDNAs and this is similarly found in the mutant.

\section{Figure 4. Binding assays for PPR_65 with RNA target sequences of ccmFeU103PS and} ccmFeU122SF. A. RNA sequence templates surrounding editing sites ccmFCeU103PS and ccmFCeU122SF as shown on top were 3' labelled with fluorescein for detection in REMSAs (RNA electrophoretic mobility shift assays). Different versions with or without C-to-U conversion at ccmFCeU103PS were used alternatively for the ccmFCeU122SF targets. Cytidines to be edited are indicated in bold, editing site position ccmFeU103PS in target ccmFeU122SF is highlighted in yellow. B. REMSAs were performed with increasing concentrations of MBP-DYW-PPR_65 $(0.1-0.4 \mu \mathrm{M})$ and labeled RNAs (4 nM) C. For

This article is protected by copyright. All rights reserved. 
competition experiments, the recombinant MBP-DYW-PPR_65 was pre-incubated with different amounts of the unlabeled ccmFC103PS RNA probe (4 - $400 \mathrm{nM})$ for 10 minutes before the labelled target was added. As a negative control, the maltose binding protein (MBP, $0.4 \mu \mathrm{M})$ alone was incubated with RNA probes to check for unspecific binding. D. Bound (complex) and free RNAs from REMSAs performed with protein amounts up to $4 \mu \mathrm{M}$ were quantified used to generate the plot.

Figure 5. PPR arrays of editing factors corresponding to 11 mitochondrial editing sites. The key amino acid positions 6 and 1' shown to be most important within each PPR repeat (Barkan et al., 2012) are aligned with the upstream RNA target sequences with the respective last $\mathrm{S}$ repeat opposite the $4^{\text {th }}$ nucleotide upstream of the editing sites ( $\mathrm{C}$ underlined, bold). The exception is the particularly long PPR array of PPR_77, which matches when shifted one nucleotide upstream. Grey shading highlights P- and S-type repeats which appear to have the major role in nucleotide recognition. Green shading indicates matches of amino acids in positions 6/1' with nucleotides according to the following rules: TN: A, TD: G, NS: C, ND: U. Mismatches are shaded in red. Pyrimidines $(\mathrm{C}, \mathrm{U})$ are less well distinguished than purines $(\mathrm{A}, \mathrm{G})$. Two asparagines (NN) in positions 6 and 1' match well with pyrimidines in P-type repeats but have also been suggested to match with purines depending on additional positions in the PPR arrays (Yagi et al., 2013). Such cases, as well as positions with pyrimidine transition mismatches are shaded in yellow. The edited $U$ in the ccmFCeU122SF target sequence is underlined.

Figure 6. Yeast-2-Hybrid assays for protein-protein interactions of Physcomitrella DYW-type PPR proteins PPR_65, PPR_71 and PPR_79 and with nine Arabidopsis MORF/RIP proteins.

The three Physcomitrella DYW-type PPRs (lacking the N-terminal localization signal) were cloned in fusion to the GAL4 activator domain (AD) and alternatively to the GAL4 DNAbinding domain (BD) and employed in $\mathrm{Y} 2 \mathrm{H}$ assays to detect protein-protein interactions. Shown are growth assays upon co-transformation of the AD constructs (arranged in lines) with the respective BD constructs (arranged in columns) after incubation for 10 days A. Control for growth of the yeast strains upon co-transfection on SD medium lacking tryptophan and leucine. B. Selection for protein-protein interactions on SD medium additionally lacking histidine and adenine. C. More stringent selection for protein-protein

This article is protected by copyright. All rights reserved. 
interactions on SD medium lacking histidine and adenine, additionally supplemented with $2.5 \mathrm{mM}$ 3-aminotriazol.

Figure 7. Possible RNA secondary structure affecting the $c c m F C$ transcript in the region of the two closely spaced RNA editing sites. The two editing events in $c c m F C$ transcript may be located adjacent to extended base pairing. Editing of the upstream site may destabilize the secondary structure to allow access of PPR_71. Alternatively binding of PPR_65 may occur before base pairing can be established or may resolve or destabilize the secondary structure which allows access of PPR_71 to the target sequence upstream of ccmFCeU122SF.

Figure 8. Conservation of editing sites ccmFeU103PS and ccmFeU122SF in the phylogeny of mosses. An 883 bp region of the $c c m F C$ gene comprising the two known editing sites in Physcomitrella patens and Funaria hygrometrica was investigated in 28 selected moss species representing all major clades. An underlying molecular phylogeny (Maximum Likelihood tree, assuming the GTR $+\Gamma+\mathrm{I}$ model of sequence evolution, allowing four rate categories for the $\alpha$-distribution) based on, and in full accordance with, a molecular data set described earlier (Wahrmund et al., 2010) is shown as a cladogram for the total taxon set of 30 species. Cytidines retained at the editing sites indicate a requirement for editing (1), whereas thymidines in the DNA eliminate the need for C-to-U RNA editing (0). A simultaneous loss of both editing sites has apparently occurred independently in basal lineages (open squares: Sphagnales, Andreaeales, Polytrichales). The loss of only the upstream editing site ccmFCeU103PS has occurred at least twice (open circles: in Bryanae and in Timmia) whereas vice versa an exclusive C-to-T conversion of editing site ccmFCeU122SF was observed only in Diphyscium (open triangle).

Figure 9. REMSA binding assay for the atp9eU92SL editing site and PPR_98. An RNA sequence target of 32 nucleotides containing the atp9eU92SL editing site (bold, underlined) was tested for binding of PPR_98. RNA electrophoretic mobility shift assays were performed with increasing concentrations of MBP-PPR_98 $(0.1-4 \mu \mathrm{M})$ and labelled RNAs (4 nM). For competitor experiments the recombinant PPR_98 was pre-incubated with increasing amounts

This article is protected by copyright. All rights reserved. 
of the unlabelled RNA probe $(4-400 \mathrm{nM})$ for 10 minutes before the labelled target was added. As negative controls, the maltose binding protein (MBP, $4 \mu \mathrm{m})$ alone was incubated with the atp9eU92SL RNA probe and, vice versa, the recombinant PPR_98 was tested for a biding to the unrelated RNA target sequence including the ccmFCeU103PS editing site.

\section{Supplementary Figure 1. Quantitative RT-PCR analysis of mitochondrial RNAs.}

P. patens wild type and KO_PPR_65 RNA from gametophores were analyzed by quantitative RT-PCR using primers covering six different mitochondrial transcripts including $c c m F C$. The KO_PPR_65 mutant was compared to the wild type plants grown in parallel on Knop supplemented with $1 \%$ sucrose and quantity of transcript is calculated in relation to the wild type amount (1). The values are means of three technical replicates (error bars indicate one standard deviation). Nuclear 18S rRNA and thioredoxin mRNA (Phypa_173694) were used as internal controls for standardization. In the mutant, amounts of transcripts of all mitochondrial genes including $c c m F C$ investigated in this study are higher in comparison to the wild type. $\mathrm{RQ}=$ relative quantity.

\section{Supplementary Figure 2. Poisoned primer extension (PPE) assay to detect putative low editing of ccmFeU122SF.}

PPE assays were conducted on the editing sites ccmFCeU103PS, ccmFCeU122SF and, as a control, on the partially edited rps14eU137SL. RT-PCR products surrounding the editing sites were obtained from WT and KO of PPR_65 and used as template for the PPE assay with 5'-FAM labelled primers, which anneal close to the editing site. The extension was stopped by incorporation of 2',3'-dideoxyguanosine-5'-triphosphate at the unedited C position producing a short product (UE, open triangle) or at the next $\mathrm{C}$ for edited molecules resulting in a longer product (E, filled triangle). As negative control, genomic DNA (gDNA) was used as template.

This article is protected by copyright. All rights reserved. 
Figure 1

A.

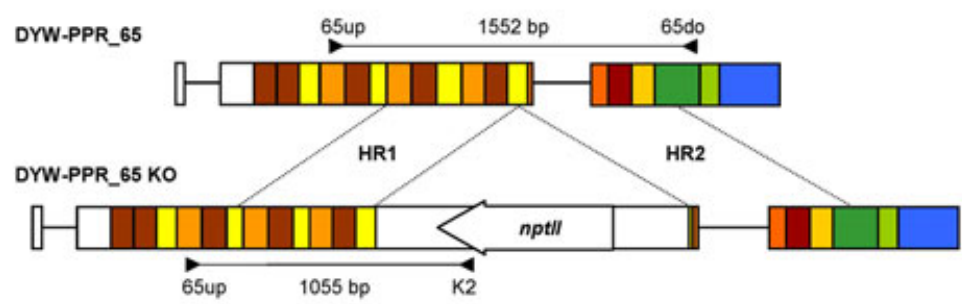

B.
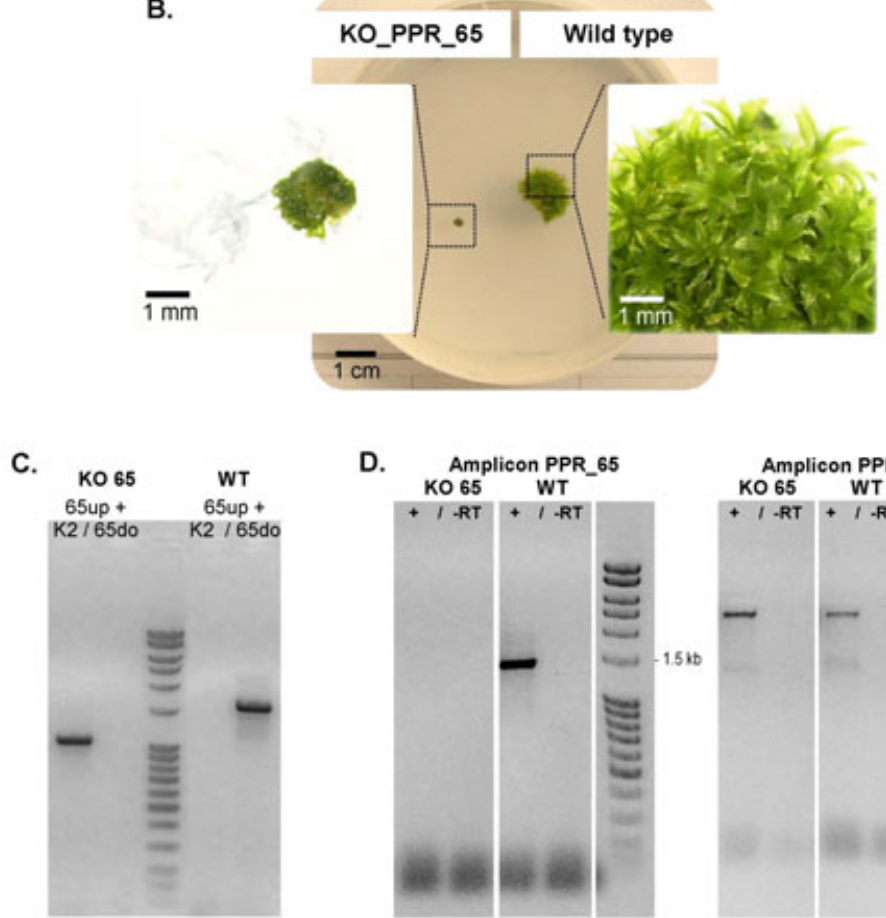

D. Amplicon PPR_65

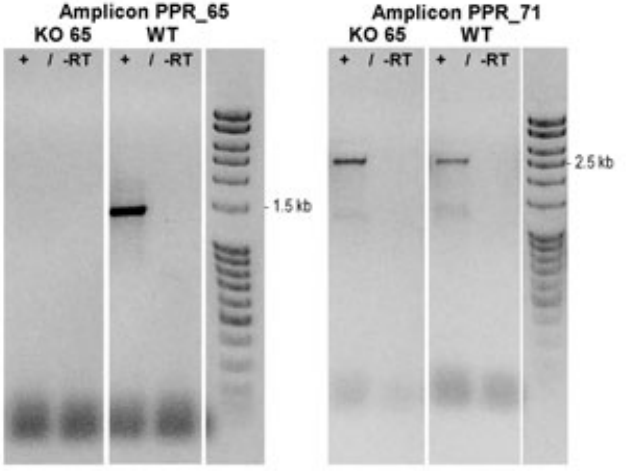

This article is protected by copyright. All rights reserved. 
Figure 2

A.
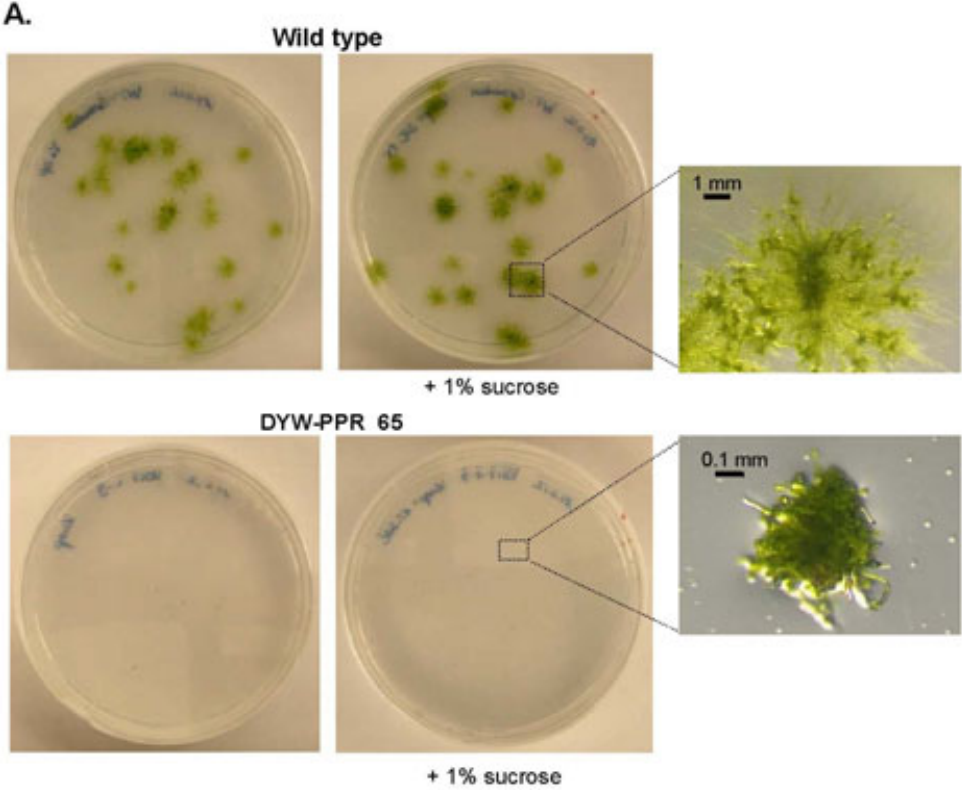

DYW-PPR_65

B.

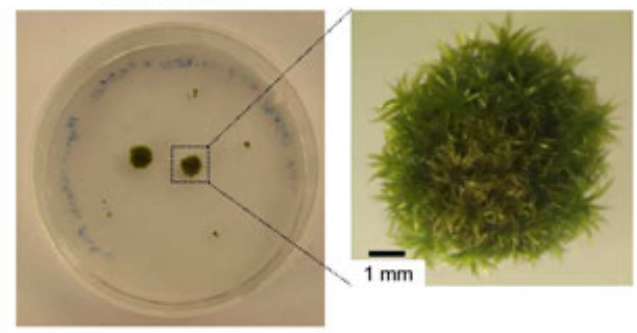

This article is protected by copyright. All rights reserved. 
Figure 3

A.
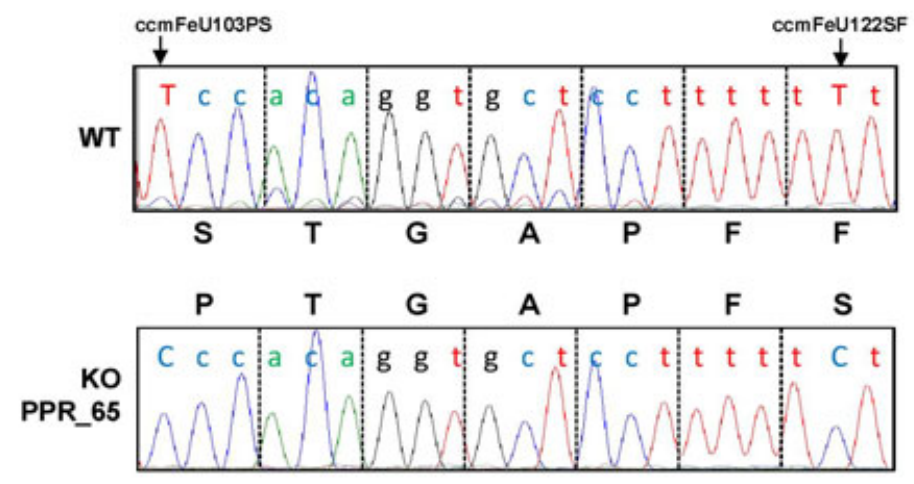

B.

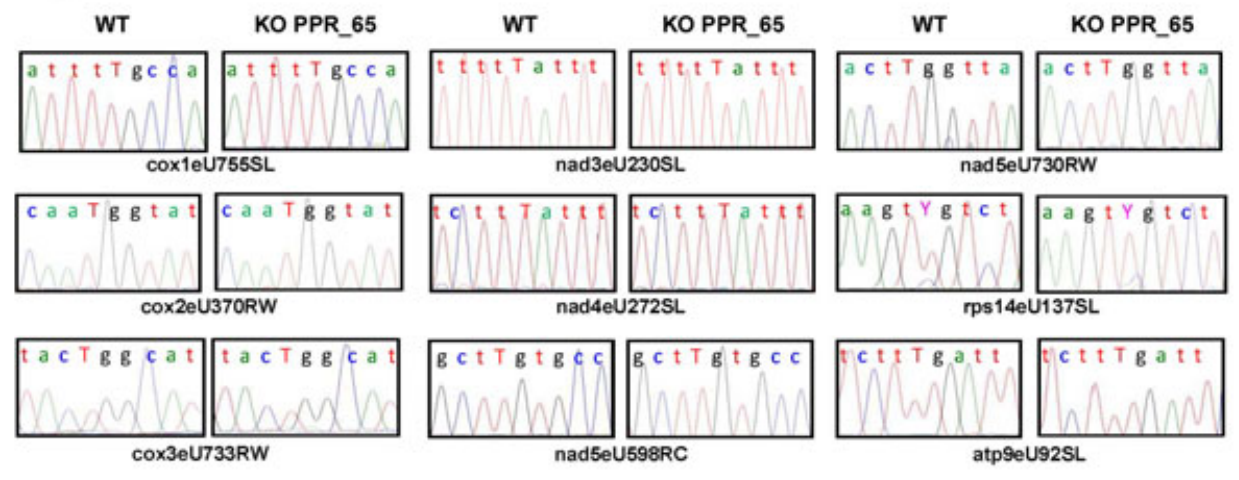

This article is protected by copyright. All rights reserved. 


\section{Figure 4}

A.

5-AAUGGUUGGUAAGUAGAGAUGUUCCCA-3-fluorescein

5-UGUUUCCACAGGUGCUCCUUUUUCUCA-3'- fluorescein

5-UGUUCCCACAGGUGCUCCUUUUUCUCA-3'- fluorescein

ccmFCeU103PS target

ccmFCeU122SF target

ccmFCeU122SF target 2

B.



c.

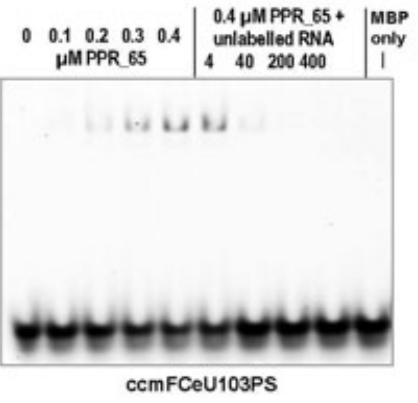

D.

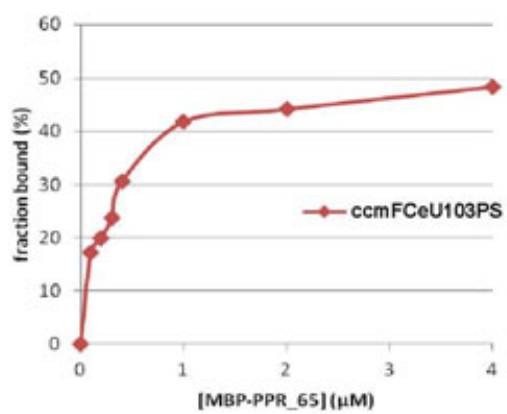

This article is protected by copyright. All rights reserved. 
Figure 5

\begin{tabular}{|c|}
\hline A. PPR_65 / Phypa_175641 \\
\hline 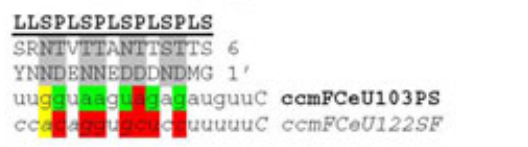 \\
\hline C. PPR_56 / Phypa_169414 \\
\hline 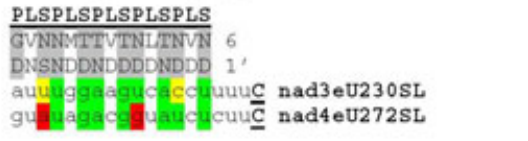 \\
\hline E. PPR_78 / Phypa_192620 \\
\hline 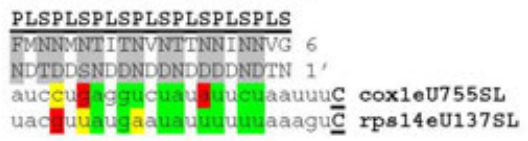 \\
\hline G. PPR_91 / Phypa_211228 \\
\hline 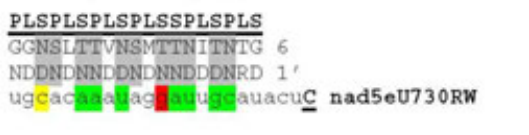 \\
\hline
\end{tabular}

B. PPR_71 / Phypa_181369

LSPLSPLSPLSPLSPLS

VNNTINLTTMTSINNVG 6

NTSDDNDDDDDDEDNTT I

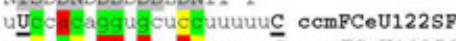



D. PPR_77 / Phypa_191892

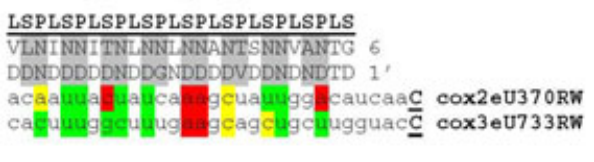

F. PPR_79 / Phypa_192787

LSPLSPLSPLSPLSPLS

VNIVNTMTNTNTMNNNVA 6

NISDDSDDDDDNDNTS 1'
acurumuct

H. PPR_98 / Phypa_224041

PLSPLSPLSPLSPLSPLSPLS

NSNNVTIVTTMTNINNITTVG 6

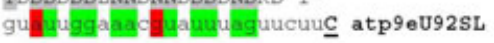

This article is protected by copyright. All rights reserved. 
Figure 6

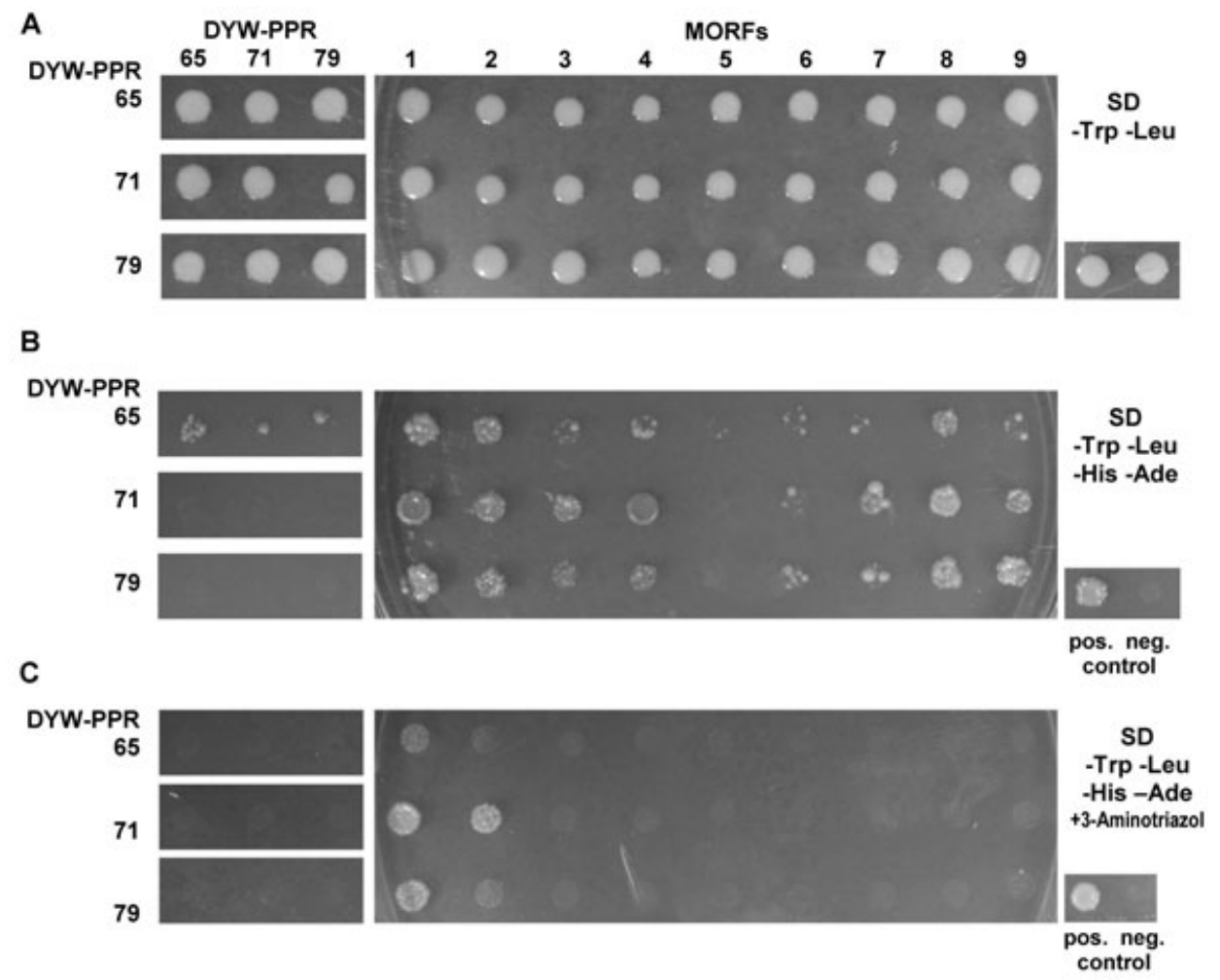

This article is protected by copyright. All rights reserved. 


\section{Figure 7}

A.
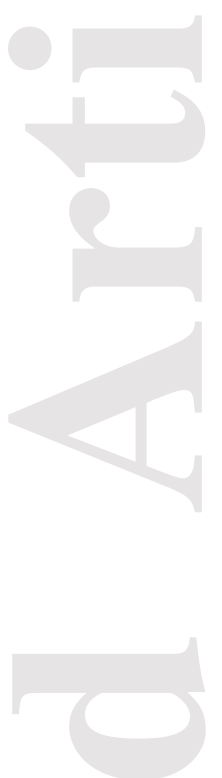

$A-A-U-G-G-U-U-A-U-G$

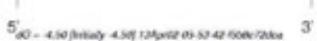

B. $5-A$

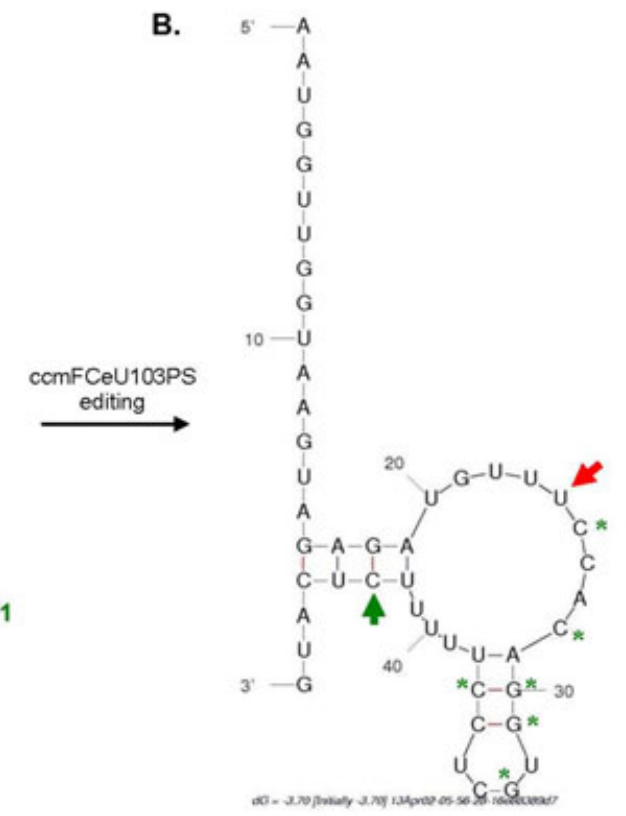

This article is protected by copyright. All rights reserved. 
Figure 8

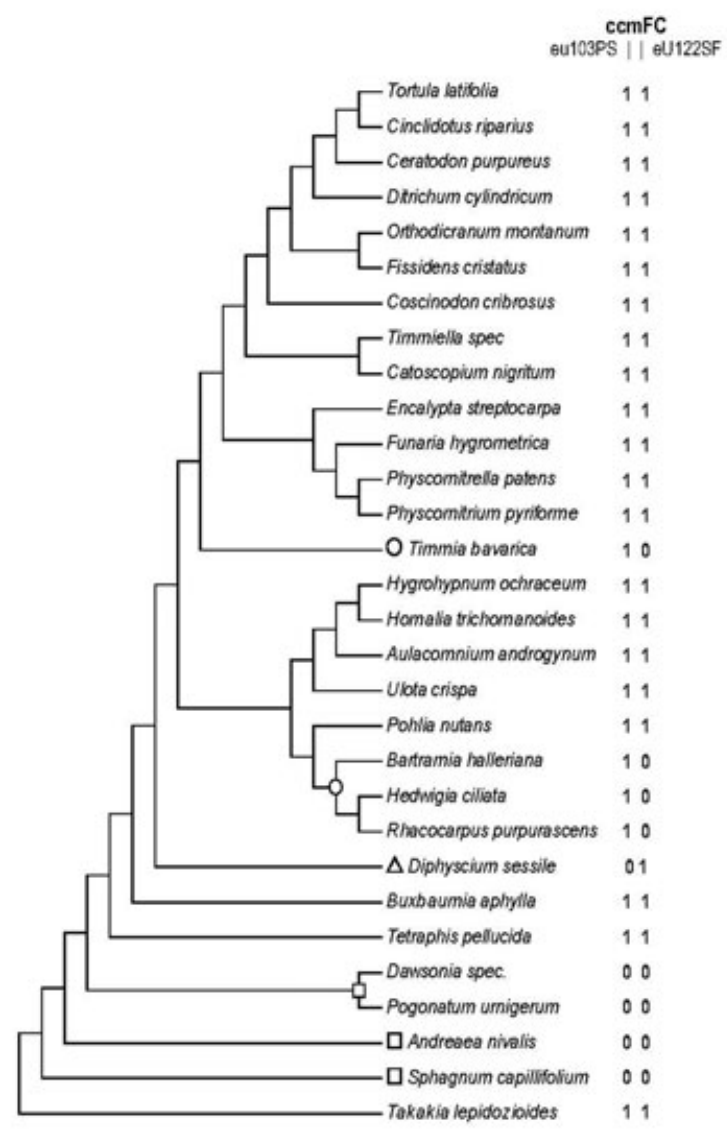

This article is protected by copyright. All rights reserved. 


\section{Figure 9}

5'-AUAGGUAUUGGAAACGUAUUUAGUUCUUCGAU-3'-fluorescein atp9eU92sL target

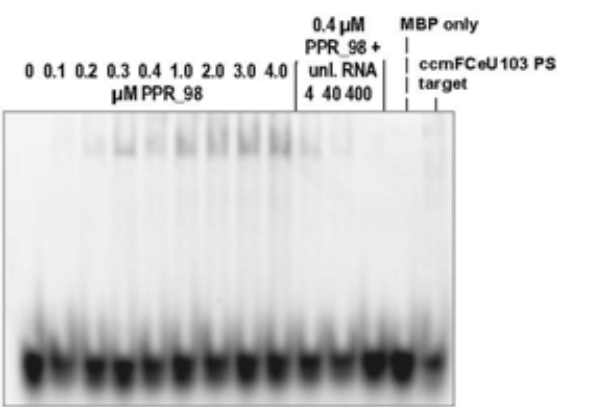

This article is protected by copyright. All rights reserved. 